\title{
Reducing Magneto-Inductive Positioning Errors in a Metal-Rich Indoor Environment
}

\author{
Orfeas Kypris, Traian E. Abrudan and Andrew Markham \\ Department of Computer Science, \\ University of Oxford, \\ Oxford, U.K. OX13QD \\ Email: firstname.lastname@cs.ox.ac.uk
}

\begin{abstract}
Ferrous objects distort magnetic fields and can significantly increase magneto-inductive positioning errors in indoor environments. In this work, we use image theory in order to formulate an analytical channel model for the magnetic field of a quasi-static magnetic dipole positioned above a perfectly conducting half-space. The proposed model can be used to compensate for the distorting effects that metallic reinforcement bars (rebars) impose on the magnetic field of a magneto-inductive transmitter node in an indoor environment. Good agreement is observed between the analytical solution and numerical solutions obtained from 2-D finite element simulations when the transmitter node is located more than $0.4 \mathrm{~m}$ above the distorters. Experimental results indicate that the image theory model shows significant improvement over the free space dipole model in estimating position along the normal to the plane of the rebars, typically reducing positioning errors by $36 \%$ in $90 \%$ of the cases.
\end{abstract}

\section{INTRODUCTION}

Indoor positioning technologies are a current subject of intensive research both in academia and industry as they enable higher level services, such as indoor navigation [1], locationbased services [2], first responder applications [3] and fall monitoring [4]. Magneto-induction is an attractive modality for positioning applications due to the fact that low-frequency magnetic fields penetrate most objects and do not suffer from multipath, which is not the case for higher frequency signals, such as WiFi. Magneto-inductive systems have previously been demonstrated in various applications, such as underground animal tracking [5], and they work particularly well when the surrounding space in the vicinity of the transmitter (TX) is void of ferrous objects [6] (Fig. II). However, when the TX is placed on the floor of a building with metallic rebars embedded within the concrete (Fig. II), the vertical ( $z$ ) component of the magnetic field vector is perturbed, which in turn invalidates the free space channel model [7]. This results to an increase in the relative position error, lowering the overall accuracy of the system.

To solve this challenge, we formulate a new channel model using image theory [8], which assumes a homogenous, solid perfect electric conductor (PEC) occupying the space from $z=0$ to $z=-\infty$. While in reality we are unlikely to operate above a solid metallic sheet, we show that our model holds well when the TX is positioned above a sparse array of metallic rebars. To validate the model, numerical simulations using COMSOL multiphysics were carried out in 2-D, and the percentage error between the spatial norm of real component of the magnetic flux density $\mathbf{B}$ was plotted as a function of position in the $y-z$ plane, where $y \subset \mathcal{P}$ and $z \perp \mathcal{P}$, where $\mathcal{P}$ is the plane of the rebars. Experimental results demonstrate significant improvement of the position estimate in $z$-axis

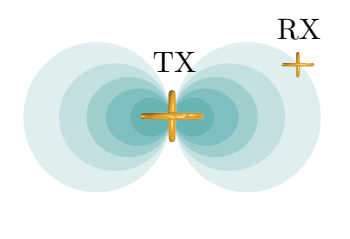

(a)

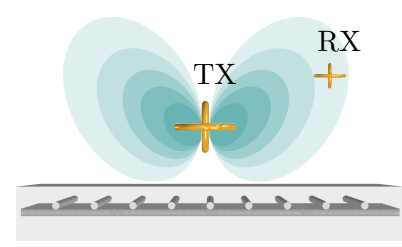

(b)
Fig. 1. Representation of the magnetic field distribution of a magnetoinductive positioning system in 2-D, depicting a triaxial coil TX and RX a) in free space, and b) over an array of rebars embedded in concrete, which perturb the field symmetry.

when using the image theory channel model, as opposed to using the free space channel model.

\section{Background on Magnetic Positioning}

Magneto-inductive positioning systems use coils to generate and detect low-frequency magnetic fields. If the physical channel model (i.e. magnetic field distribution) of the system is known, one can obtain position estimates of the receiver (RX) w.r.t. the TX, by optimizing the model against the measured data and extracting the position vector. A position estimate in $\mathbb{R}^{\mathrm{n}}$ requires $n$ mutually orthogonal coils. Thus, in 2-D one would only need biaxial units, while in 3-D triaxial units are required. In contrast, range based systems require $n+1$ noncolocated transmitters.

\section{A. Magnetic field in free space}

At low enough frequencies, $k a \ll 1$, where $k$ is the wavenumber and $a$ is the radius of the coil. This condition makes it possible to treat the TX as an infinitesimal (point) magnetic dipole operating in the near field. For reasons of mathematical tractability we consider the 2-D case; extension to 3-D can easily be obtained subsequently. In free space, where conducting and/or ferromagnetic objects are absent (Fig. II), the magnetic flux density of the point dipole positioned at the origin of the world coordinate frame takes on the following form [7]:

$$
\mathbf{B}(\mathbf{r}, \mathbf{m})=\frac{\mu_{T X}}{2 \pi\|\mathbf{r}\|^{2}}\left(\frac{2 \mathbf{r r}^{T}}{\|\mathbf{r}\|^{2}}-\mathbf{I}_{2}\right) \mathbf{m}
$$

where $\mathbf{B}(\mathbf{r}, \mathbf{m})$ is the magnetic flux density at the position vector $\mathbf{r}$ due to magnetic dipole moment $\mathbf{m}, \mathbf{I}_{2}$ is the $2 \times 2$ identity matrix, and $\mu_{T X}$ is the relative permeability of the TX coil, which in our study we consider to be equal to the permeability of free space $\left(4 \pi \times 10^{-7} \mathrm{H} / \mathrm{m}\right)$. Using (1), it 
is possible to recover the 2-D position of TX w.r.t RX, from measurements of the 2-D magnetic field vector measured at the RX position, in free space.

\section{B. Magnetic field in the vicinity of a conducting object}

In the vicinity of conducting objects the free space model of (1) is no longer valid (Fig. II). The field generated by the TX induces eddy currents within the conductors, which then radiate a secondary (scattered) field. The total field $\mathbf{B}^{t}$ outside the conducting regions will then be equal to $\mathbf{B}^{\mathrm{t}}=\mathbf{B}^{\mathrm{inc}}+\mathbf{B}^{\mathrm{s}}$, where $\mathbf{B}^{\text {inc }}$ is the incident field due to the point dipole as in (1), and $\mathbf{B}^{\mathrm{s}}$ is the scattered field due to re-radiation by the conducting objects. Under the assumption that the objects are perfectly conducting, larger than one skin depth, and densely positioned on the plane $z=0$ in a Cartesian coordinate system, they can be approximated by an infinitely large electric ground plane that coincides with plane $\mathcal{P}$ (Fig. $2)$. At the interface between free space and a perfect electric conductor (PEC), the tangential component of the electric field is continuous, such that $\mathbf{z} \times \mathbf{E}=0$. To solve for the total field, there are two alternatives. We can either 1) solve Maxwell's equations in the half-space above the PEC plane taking into account the aforementioned boundary condition, or 2) replace the PEC with free space and place an image dipole at $z=-h$, which will satisfy the aforementioned boundary condition at $z=0$. In the latter case the total field $\mathbf{B}^{\mathrm{t}}$ will be a sum of the dipole and image dipole fields, such that:

$$
\begin{aligned}
\mathbf{B}^{\mathrm{t}} & =\frac{\mu_{T X}}{2 \pi}\left[\left(\frac{1}{\left\|\mathbf{r}_{1}\right\|^{2}}\right)\left(\frac{2 \mathbf{r}_{1} \mathbf{r}_{1}^{T}}{\left\|\mathbf{r}_{1}\right\|^{2}}-\mathbf{I}_{2}\right)\right. \\
& \left.+\left(\frac{1}{\left\|\mathbf{r}_{2}\right\|^{2}}\right)\left(\frac{2 \mathbf{r}_{2} \mathbf{r}_{2}^{T}}{\left\|\mathbf{r}_{2}\right\|^{2}}-\mathbf{I}_{2}\right) \mathbf{M}_{z}\right] \mathbf{m}
\end{aligned}
$$

where $\mathbf{r}_{1}=\left[y_{r}, z_{r}+h\right]^{T}, \mathbf{r}_{2}=\left[y_{r}, z_{r}-h\right]^{T}$ and $y_{r}, z_{r}$ are the $y$ - and $z$ coordinates of the RX. $\mathbf{M}_{z}$ denotes a reflection matrix about plane $\mathcal{P}$ (Fig. 2). The above expression is only valid in the region $z>0$, since inside a PEC, $\mathbf{E}=0$, which also implies it cannot support an alternating magnetic field. In the previous expressions it is implicit that the TX orientation $\Theta$ and receiver orientation $\Omega$ are identical and aligned with the world frame, such that $\boldsymbol{\Theta}=\boldsymbol{\Omega}=\mathbf{I}_{\mathbf{2}}$, where $\mathbf{I}_{\mathbf{2}}$ is a $2 \times 2$ identity matrix.

\section{Derivation of the channel model}

In previous work [7], an eigenvalue decomposition method was derived in order to obtain the position of a TX in free space, using vector measurements of the magnetic flux density at the receiver position. The same method, however, cannot be applied to the present analytical expression of (2), as crossmultiplication terms prevent factorization into separate range and orientation components. To obtain the magnetic vector field of the TX but in the receiver frame, we need to multiply by $\boldsymbol{\Omega}^{T}$, such that:

$$
\begin{aligned}
\boldsymbol{\Omega}^{T} \mathbf{B}^{\mathrm{t}} & =\frac{\mu_{T X}}{2 \pi} \boldsymbol{\Omega}^{T}\left[\left(\frac{1}{\left\|\mathbf{r}_{1}\right\|^{2}}\right)\left(\frac{2 \mathbf{r}_{1} \mathbf{r}_{1}^{T}}{\left\|\mathbf{r}_{1}\right\|^{2}}-\mathbf{I}_{2}\right)\right. \\
& \left.+\left(\frac{1}{\left\|\mathbf{r}_{2}\right\|^{2}}\right)\left(\frac{2 \mathbf{r}_{2} \mathbf{r}_{2}^{T}}{\left\|\mathbf{r}_{2}\right\|^{2}}-\mathbf{I}_{2}\right) \mathbf{M}_{y}\right] \underbrace{\left[\mathbf{e}_{1}, \mathbf{e}_{2}\right]}_{\mathbf{I}_{2}}
\end{aligned}
$$

where $\mathbf{e}_{1}$ and $\mathbf{e}_{2}$ are unit excitation vectors along $y-$ and $z$ axes. We can define the $2 \times 2$ MIMO channel matrix:

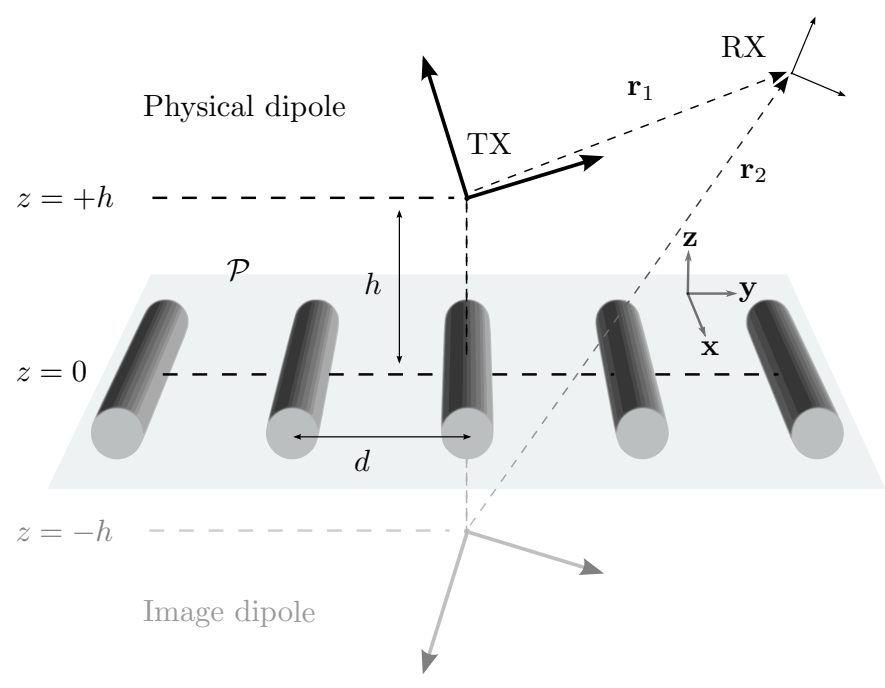

Fig. 2. Illustration of the problem, where the TX is positioned at some height $z=+h$ with its corresponding image positioned at $z=-h$. The position vectors of the RX w.r.t the TX and its image are $\mathbf{r}_{1}$ and $\mathbf{r}_{2}$ respectively. The distance between the geometric centers of the rebars is $d$. The basis coordinate system is denoted as $(\mathbf{x}, \mathbf{y}, \mathbf{z})$.

$\mathbf{S}=c \boldsymbol{\Omega}^{T} \mathbf{B}^{\mathrm{t}}$, where $c$ is a range-dependent scaling factor $\left(c \propto r^{-2}\right.$ in 2-D and $c \propto r^{-3}$ in 3-D), which also incorporates the TX/RX coils specific parameters (area, no. of turns, amplifier gain) and can be determined by calibration from a single measurement taken at a known distance [7].

\section{SIMULATION}

Numerical solutions for the magnetic field in the presence of steel reinforcement bars were obtained using the AC/DC module within the COMSOL Multiphysics software package. The problem is treated as quasi-static and Ampere's law is used to obtain the magnetic field $\mathbf{H}$ in all regions. In free space, the propagation constant $k=\omega \sqrt{\mu_{0} \epsilon_{0}}$, and in steel, $k=\omega \sqrt{\mu \epsilon}$, where both the magnetic permeability $\mu$ and electrical permittivity $\epsilon$ are complex quantities, and are defined as $\mu=\mu_{0}\left(\mu_{r}^{\prime}-j \mu_{r}^{\prime \prime}\right)$ and $\epsilon=\epsilon_{0}\left(\epsilon_{r}^{\prime}-j \epsilon_{r}^{\prime \prime}\right)$, with $\epsilon_{r}^{\prime \prime}=\sigma / \omega \epsilon_{0}$, where $\sigma$ and $\omega$ are the electrical conductivity and angular frequency respectively. To simplify the problem, we assume that the magnetic permeability of steel is real and linear. Ignoring the imaginary part is a reasonable assumption when operating at low frequencies, and assuming a linear permeability is reasonable when operating close to the origin of the $B-H$ curve and below magnetic saturation, which for typical construction steels ranges from 1.0 to $2.0 \mathrm{~T}$. Similarly, it is reasonable to ignore the real part of the complex permittivity, as in the case of conductors $\epsilon_{r}^{\prime \prime} \gg \epsilon_{r}^{\prime}$, owing to their high value of electrical conductivity. For this simulation, $\sigma=1.12 \times 7$ $\mathrm{S} / \mathrm{m}$ and $\mu_{r}=4000$. COMSOL implements the appropriate electromagnetic boundary conditions at the interface between media. To ensure that $\mathbf{H} \rightarrow 0$ as $\mathbf{r}_{1}, \mathbf{r}_{2} \rightarrow \infty$, infinite elements were used at the outer boundary of the solution region. To minimize boundary effects even further, the outer boundary was placed at a distance approximately 5 times the width of the region of interest ( 3 meter radius from the TX). The mesh was fine-tuned to a maximum element size of $0.05 \mathrm{~m}$ within the region of interest, in order to avoid discretization errors arising from high field gradients, and was progressively 

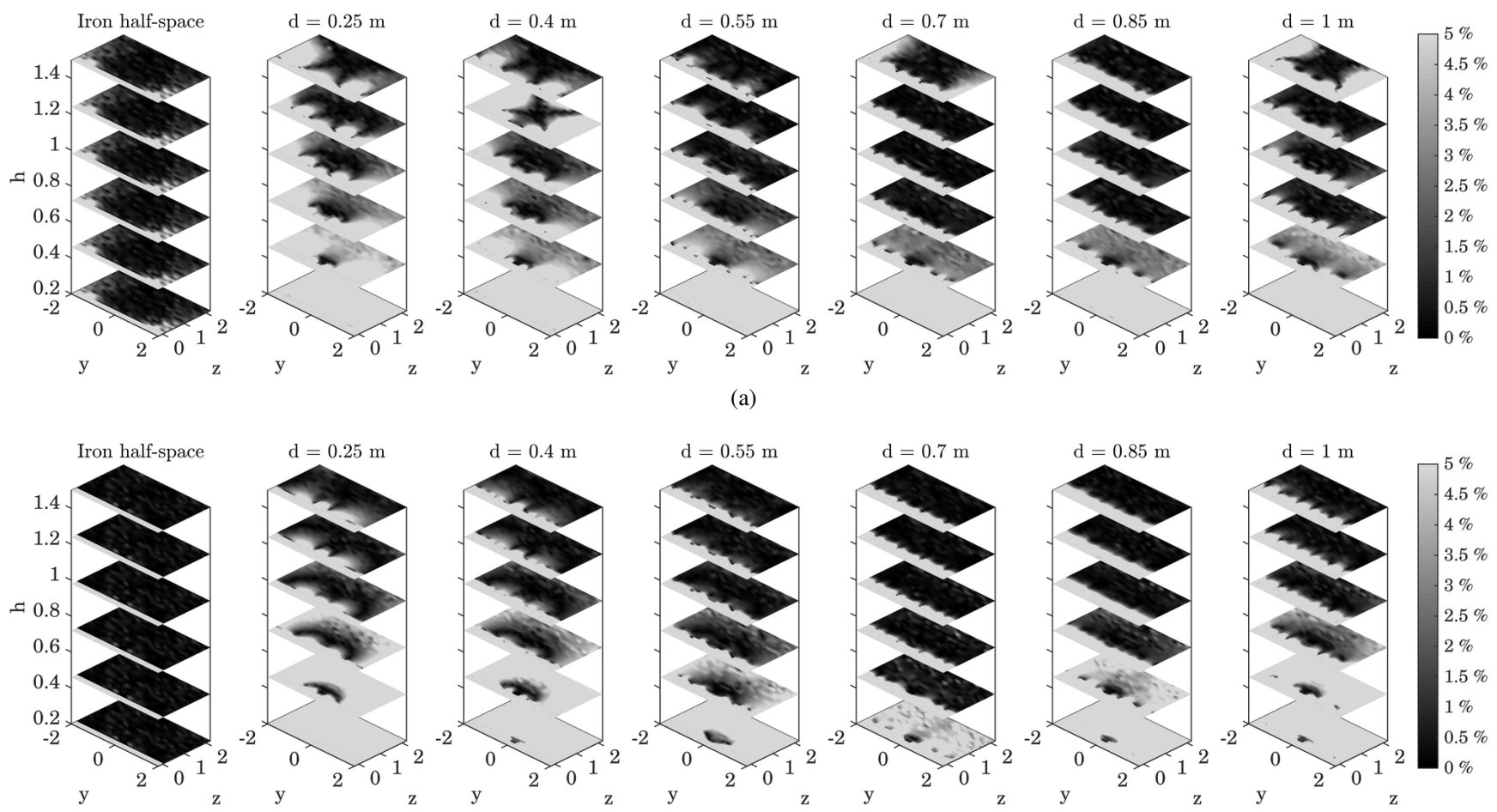

(b)

Fig. 3. Percentage error in $\|\mathbf{B}\|$ between analytical model and COMSOL simulation for the (a) $\hat{\mathbf{y}}$-oriented and (b) (a) $\hat{\mathbf{z}}$-oriented TX dipoles, where $x$, $y$ and $h$ denote the $\mathrm{x}$ - and y-coordinates as well as the vertical TX distance from the plane of the iron half-space and the rebars, and $d$ denotes the spacing between the geometric centers of the rebars. Frequency of the magnetic dipoles was set to $2500 \mathrm{~Hz}$.

coarsened towards the outer boundary.

The simulation was parametrized to run for different combinations of $h$ and $d$. Additionally, each one of those combinations was solved twice, one for each axis excitation $\mathbf{e}_{i}$, similarly to how it would be done in a practical situation, where the TX axes are sequentially energized. An $m \times n$ array of channel matrices was then assembled for each simulation, where $m \times n$ is the grid of $y-z$ points ported to the localization algorithm. It should be noted that even though eddy currents are induced in the conducting objects, their magnitude is small enough that they can be ignored. Solely the real part of the field was considered.

\section{RESUlts AND Discussion}

\section{A. Simulation}

The COMSOL model solutions for the real component of the magnetic flux density $\mathbf{B}^{\mathrm{t}}$ were extracted and the spatial norm of the flux density vector $\left\|\mathbf{B}^{\mathrm{t}}\right\|=\sqrt{B_{y}^{2}+B_{z}^{2}}$ was computed. In order to compare the numerical solution to the analytical image theory model of (2) an error metric of the following form was used:

$$
\operatorname{err}=\frac{\left\|\mathbf{B}_{\mathrm{num}}^{\mathrm{t}}\right\|-\left\|\mathbf{B}_{\mathrm{an}}^{\mathrm{t}}\right\|}{\left\|\mathbf{B}_{\mathrm{num}}^{\mathrm{t}}\right\|} \cdot 100[\%]
$$

where $\mathbf{B}_{\text {num }}^{\mathrm{t}}$ and $\mathbf{B}_{\mathrm{an}}^{\mathrm{t}}$ denote the spatial norm of the real component of $\mathbf{B}^{\mathrm{t}}$ obtained from numerical and analytical solutions respectively. Figs. $3 \mathrm{a}$ and $3 \mathrm{~b}$ illustrate this error metric in the $y-z$ plane for the $\mathbf{y}$ - and $\mathbf{z}$-oriented TX dipoles respectively, for a variety of TX heights $h$ and rebar spacings $d$, as well as for the case where the reflecting surface is a homogeneous half-space composed of steel.

In the case of the iron half-space, the error metric of (4) for all TX positions is significantly smaller than $5 \%$, which indicates good agreement with the analytical model of (2). The introduction of rebars increases the discrepancy between numerical and analytical solutions for TX positions $h<0.8$, and especially for $h<0.2$ (where the error exceeds $5 \%$ ), as the inclusion of finite geometries invalidates the assumptions utilized by the method of images. Furthermore, on average it can be seen that for the $\mathbf{y}$-oriented dipole the average error decreases monotonically with increasing $d$, while for the $\mathbf{z}$ oriented dipole there is a critical spacing $(d=0.7 \mathrm{~m})$ at which the error drops. This is a frequency- and geometry-dependent near-field phenomenon which will be addressed in future work. It is possible to obtain an estimate of parameters $x, y, h$ by formulating a cost function subject to the following constraints:

$$
\{\hat{x}, \hat{y}, \hat{z}\}=\underset{x, y, h}{\arg \min }\left\|\mathbf{S}_{\mathrm{num}}-\mathbf{S}_{\mathrm{an}}\right\|_{\mathrm{F}}
$$

where subscript $\mathrm{F}$ denotes the Frobenius norm, $\mathbf{S}_{\text {num }}=c \mathbf{B}_{\text {num }}^{\mathrm{t}}$ and $\mathbf{S}_{\mathrm{an}}=c \mathbf{B}_{\mathrm{an}}^{\mathrm{t}}$ (since $\boldsymbol{\Omega}=\mathbf{I}_{2}$ ).

\section{B. Experiment}

In previous work [7] where the model of (1) was utilized, the position vector was found to be the eigenvector corresponding to the maximal eigenvalue of the Gram matrix $\mathbf{C}=\mathbf{S}^{T} \mathbf{S}$. The distortions in the field due to nearby ferrous objects caused the position vector to undergo a $90^{\circ}$ rotation, 


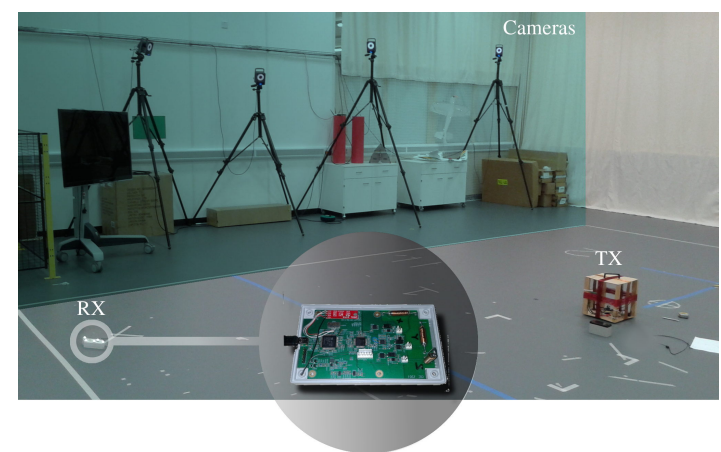

Fig. 4. Measurement area, depicting the TX and RX, as well as the system of cameras used to obtain ground truth.

equivalent to swapping the $x$ and $y$ axes. We corrected this ambiguity manually after running the optimization algorithm (usage of two transmitter would not require manual correction). Hemispherical ambiguity (which is a known challenge of MI positioning) had to be corrected manually as well.

In order to verify the validity of our model, we collected a large number of indoor magneto-inductive measurements in a large hall whose floor contains rebars of unknown geometry and material properties. The measurement area is shown in Fig. 4. TX was placed on the floor, whereas RX was subsequently placed at 1000 different locations enclosed in a volume of $6 \times 8 \times 1.3$ meters. TX and RX orientations were the same as the global coordinate frame, i.e., $\boldsymbol{\Omega}=\boldsymbol{\Theta}=\mathbf{I}_{2}$. Millimeter accuracy ground truth for the RX positions was provided by system of 8 Vicon cameras placed around the measurement area. To obtain the estimates, the models of (1) and (2) were modified to account for measurements in 3-D [7]. The error estimate in the $\mathrm{y}$-direction was calculated as follows:

$$
e_{y}=|| \hat{y}_{r}|-| y_{r}||
$$

where $\hat{y}_{r}$ is the estimated position of the receiver along $y$. The image theory model results exhibited abnormally high values for $z$ and $h$, which were nevertheless significantly close to each other in most cases. This can be explained as follows: the theory assumes a homogeneous conducting half-space which significantly distorts the magnetic field of a dipole placed in its vicinity. To reduce the distortion in the field, one could either place the dipole further away from the half-space, or substitute the half-space by a sparse grid of rebars underneath the dipole, provided the distance between them is not too close (see previous section). Furthermore, the values of $z$ and $h$ fluctuated (while preserving their difference on average), which may indicate regions where the rebar arrangement is aperiodic. Thus, to normalize for the effect of an unknown amount of metal, at an unknown distance underneath the dipole, the error estimate in the z-direction was calculated as follows:

$$
e_{z}=|| \hat{z_{r}}|-| \hat{h}||-|| z_{r}|-| h||,
$$

where $\hat{z_{r}}$ is the estimated position of the receiver along $z$, and for our measurements $h=0.17 \mathrm{~m}$. In the case of the free space model, $h=\hat{h}=0$, as the model only optimizes for $y$ and $z$. The results of Fig. 5a validate each other as they are practically identical, however Fig. 5b shows that by using the

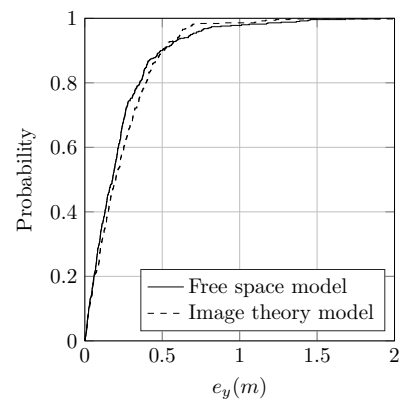

(a)

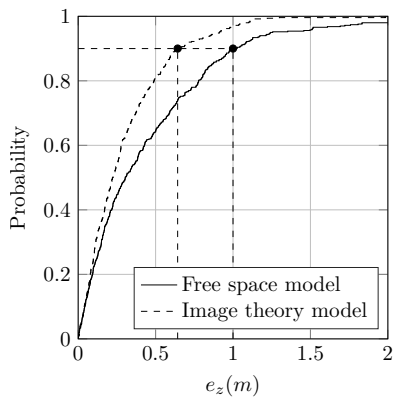

(b)
Fig. 5. Errors in the $y$ - and $z$ position estimates computed from (6) and (7). Error in the $z$ etimate can be seen to improve significantly by using the image theory model.

image theory model we obtain $36 \%$ smaller positioning error in $90 \%$ of the cases.

\section{CONCLUSion}

In this work, we derived and experimentally verified a new magneto-inductive channel model that reduces positioning errors in a metal-rich indoor environment. Compared to the free-space model, our model reduces positioning errors in $z$ by $\sim 36 \%$ in $90 \%$ of the cases. This enables applications which require sub-meter positioning accuracy (such as drone position estimation and fall detection).

\section{ACKNOWLEDGMENT}

The authors would like to thank EPSRC for funding this research (Grant ref. EP/L00416X/1 Digital Personhood: Being There: Humans and Robots in Public Spaces (HARPS), and Grant ref. EP/M017583/1 Magneto-Inductive Six Degree of Freedom Smart Sensors (MiSixthSense) for Structural and Ground Health Monitoring), and Dr. Paul Bremner from the Bristol Robotics Laboratory for providing the camera-based measurement facilities (Vicon).

\section{REFERENCES}

[1] R. Mautz, "Overview of current indoor positioning systems," Geodesy and Cartography, vol. 35, no. 1, pp. 18-22, 2009.

[2] D. Mohapatra and S. Suma, "Survey of location based wireless services," in IEEE ICPWC, Jan 2005, pp. 358-362.

[3] J. Rantakokko, J. Rydell, P. Stromback, P. Handel, J. Callmer, D. Tornqvist, F. Gustafsson, M. Jobs, and M. Gruden, "Accurate and reliable soldier and first responder indoor positioning: multisensor systems and cooperative localization," Wireless Commun., IEEE, vol. 18, no. 2, pp. 10-18, April 2011.

[4] T. Shany, S. J. Redmond, M. R. Narayanan, and N. H. Lovell, "Sensorsbased wearable systems for monitoring of human movement and falls," IEEE Sensors J., IEEE, vol. 12, no. 3, pp. 658-670, 2012.

[5] A. Markham and N. Trigoni, "Underground localization in 3-D using magneto-inductive tracking," IEEE Sensors J., no. 6, pp. 1809-1816, June 2012.

[6] Z. Sun and I. Akyildiz, "Underground wireless communication using magnetic induction," in IEEE ICC, June 2009, pp. 1-5.

[7] T. E. Abrudan, Z. Xiao, A. Markham, and N. Trigoni, "Distortion rejecting magneto-inductive 3-D localization (MagLoc)," IEEE J. Sel. Areas Commun., vol. PP, no. 99, pp. 1-14, May 2015.

[8] J.-M. Jin, Theory and computation of electromagnetic fields. Hoboken and N.J: Wiley, 2010 